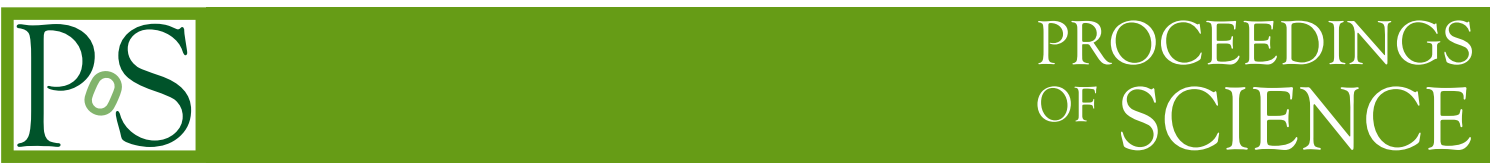

\title{
Confining properties of two-color QCD at finite density
}

\section{Simone Conradi}

Università di Genova \& INFN

E-mail: Simone.Conradi@ge.infn.it

Alessio D’Alessandro*

Università di Genova \& INFN

E-mail: adales@ge.infn.it

\section{Massimo D’Elia}

Università di Genova \& INFN

E-mail: delia@ge.infn.it

\begin{abstract}
A magnetically charged disorder parameter is used to detect the deconfining transition in two color QCD at finite temperature and density. We note that a finite density of baryonic matter induces the disappearing of dual superconductivity and that this phenomenon coincides with the restoration of chiral symmetry also at finite density, at last in the range of temperatures explored. The onset of the unphysical saturation transition is studied as well, and a warning is given about the possible effects that the Pauli blocking could have at strong coupling.
\end{abstract}

The XXV International Symposium on Lattice Field Theory

July 30-4 August 2007

Regensburg, Germany

${ }^{*}$ Speaker. 


\section{Introduction}

Color confinement is not yet fully understood from QCD first principles but lattice simulations have anyway provided evidence for a deconfining transition taking place where other important phenomena happen, like chiral symmetry restoration.

In this paper we study how deconfinement at finite chemical potential $\mu$ compares to what happens at $\mu=0$ and how it is related to the restoration of the chiral symmetry in the QCD phase diagram: this interplay could help clarifying the very nature of confinement. The topic has already been studied in previous literature: in particular preliminary results about deconfinement at high densities and low temperatures have been obtained in Ref. [1], an analysis in the large $N_{c}$ limit has been performed in Ref. [2], while the relationship among the chiral transition, confinement, and other observables such as the topological charge susceptibility has been investigated in Ref. [3].

However previous studies have mainly used the expectation value of the Polyakov loop to detect the deconfinement transition, a parameter not suitable for the theory with dynamical fermions, where the center symmetry to which it is related is already explicitely broken. For that reason in the present study we look for different order parameters defined in a specific model of confinement but valid also in full QCD.

The framework is that of the dual superconducting model of the QCD vacuum $[4,5,6]$, where confinement is associated to the breaking of an abelian dual symmetry and to the condensation of magnetic charges. In this context we may use the disorder parameter $\langle\mathscr{M}\rangle^{1}$ developed by the Pisa group [7,8], which consists in the expectation value of an operator that creates a magnetic monopole. $\langle\mathscr{M}\rangle$ has been shown to be a good probe for color confinement both in pure gauge [9, $10]$ and in full QCD [11, 12]: by means of this parameter we are going to look to the confining properties of the various phases in the QCD phase diagram.

Since the sign problem makes a numerical study of finite density QCD very difficult, here we restrict ourselves to QCD with two colors, where such a problem is missing because the determinant of the fermionic matrix is real. In principle no significant differences are expected.

We have simulated 8 flavours of staggered fermions of mass $\hat{m}_{q}=0.07$ using an exact HMC algorithm. We have used $L_{s} \times L_{t}$ lattices with $L_{t}=6$ and $L_{s}=8,12,16$.

A full account of our results has been published in [13].

\section{The disorder parameter $\langle\mathscr{M}\rangle$}

The magnetically charged operator $\mathscr{M}(\vec{x}, t)$ creates a magnetic monopole in $\vec{x}, t$ by shifting the field by the classical vector potential of a monopole, $\vec{b}_{\perp}(\vec{x}-\vec{y})$, and can be written [8] as $\mathscr{M}(\vec{x}, t)=$ $\exp \left[\frac{i}{e} \int d^{3} x \vec{E}_{\perp}(\vec{y}, t) \vec{b}_{\perp}(\vec{y}-\vec{x})\right]$, where the electric field $\vec{E}_{\perp}(\vec{y}, t)$ is the momentum conjugate to the quantum vector potential. Its expectation value, detecting dual superconductivity, can be expressed on the lattice as $\langle\mathscr{M}\rangle=\tilde{Z} / Z$, where $Z$ is the normal QCD partition function, while $\tilde{Z}$ is obtained from $Z$ by a change in the pure gauge action $S_{G} \rightarrow \tilde{S}_{G}$ : a monopole field is added to the temporal plaquettes at the monopole creation timeslice.

\footnotetext{
${ }^{1}$ We change the usual notation for the disorder operator, $\langle\mu\rangle$, in order to avoid confusion with the notation for the chemical potential.
} 
The numerical study of a ratio of two different partition functions such as $\langle\mathscr{M}\rangle$ is very difficult: that is why we will rather study susceptibilities of the disorder parameter, from which the behaviour of $\langle\mathscr{M}\rangle$ at the phase transition can be inferred.

For instance, if we are interested in $\langle\mathscr{M}\rangle(\beta)$, as when studying the $\mu=0$ phase transition, we look to $[7,8,9]$

$$
\rho=\frac{\partial}{\partial \beta} \ln \langle\mathscr{M}\rangle=\frac{\partial}{\partial \beta} \ln \tilde{Z}-\frac{\partial}{\partial \beta} \ln Z=\langle S\rangle_{S}-\langle\tilde{S}\rangle_{\tilde{S}}
$$

where the action used for Monte Carlo sampling is indicated by the subscript.

At finite temperature and density we are interested instead in the behaviour of $\langle\mathscr{M}\rangle$ at fixed $\beta$, i.e. fixed temperature, ${ }^{2}$ and variable $\hat{\mu} \equiv a \mu$. For that reason we look at

$$
\rho_{D} \equiv \frac{\partial}{\partial \hat{\mu}} \ln \langle\mathscr{M}\rangle=\frac{\partial \ln \tilde{Z}}{\partial \hat{\mu}}-\frac{\partial \ln Z}{\partial \hat{\mu}}=\left\langle N_{q}\right\rangle_{\tilde{S}}-\left\langle N_{q}\right\rangle_{S}
$$

where $N_{q}$ is the quark number operator, $\left\langle N_{q}\right\rangle=\left\langle\operatorname{Tr}\left(\frac{\partial M}{\partial \hat{\mu}} \cdot M^{-1}\right)\right\rangle$, and $M$ is the fermion matrix. ${ }^{3}$

The disorder parameter can be recovered as the integral of (2.1) with the initial condition $\langle\mathscr{M}\rangle=1$ at $\beta=0$ : in particular $\langle\mathscr{M}\rangle$ abruptly falls at the phase transition if $\rho$ has here a sharp negative peak and $\langle\mathscr{M}\rangle$ is exactly zero beyond the deconfining phase transition if the peak diverges in the thermodynamical limit. A similar behavior is expected for $\rho_{D}$ if a deconfining transition is met at $\mu \neq 0$ starting from a point in the confined phase at $\mu=0$.

The two susceptibilities $\rho$ and $\rho_{D}$ do not only locate the position of the critical superconducting transition line, but also may be used to detect its tangent. Actually in the $\beta-\hat{\mu}$ plane the gradient $\vec{\nabla}\langle\mathscr{M}\rangle=\left(\frac{\partial\langle\mathscr{M}\rangle}{\partial \beta}, \frac{\partial\langle\mathscr{M}\rangle}{\partial \hat{\mu}}\right)=\left(\rho, \rho_{D}\right)\langle\mathscr{M}\rangle$, is orthogonal to the critical line, whose slope is then equal to $-\rho_{D} / \rho$.

\section{Numerical results}

\subsection{The deconfining transition at zero chemical potential}

We will first check the coincidence of the deconfining and chiral breaking transitions at $\mu=0$. In Fig. 1 [left] we show the peaks of $\chi_{c}$, the disconnected part of the chiral susceptibility, $\chi_{L}$, the Polyakov loop susceptibility, and $\chi_{P}$, the plaquette susceptibility. Our estimate for the location of the transition (from a fit to the chiral susceptibility) is $\beta_{c}=1.582(2)$, to be compared to those obtained by fitting the Polyakov loop susceptibility $\left(\beta_{L}=1.587(4)\right)$ and the plaquette susceptibility $\left(\beta_{P}=1.575(5)\right) . \rho$ (Fig. 1 [right]) displays a peak at the same coupling, hence we can state that at $\mu=0$ deconfinement and the chiral restoration coincide.

\subsection{The deconfining transition at non zero chemical potential}

At fixed $\beta$ we look to the behaviour of $\langle\mathscr{M}\rangle$ as a function of $\hat{\mu}$ by means of $\rho_{D}$. We have considered only values of $\beta$ below $\beta_{c}(\hat{\mu}=0)$, in particular $\beta=1.50$ and $\beta=1.55$ : in this case we

\footnotetext{
${ }^{2}$ We remind that $\beta$ is related to the temperature via $T=1 /\left(L_{t} a(\beta)\right)$ where $L_{t}$ is the lattice temporal extension.

${ }^{3} \mathrm{An}$ additional factor 2 is actually needed for our case with 8 staggered flavors.
} 

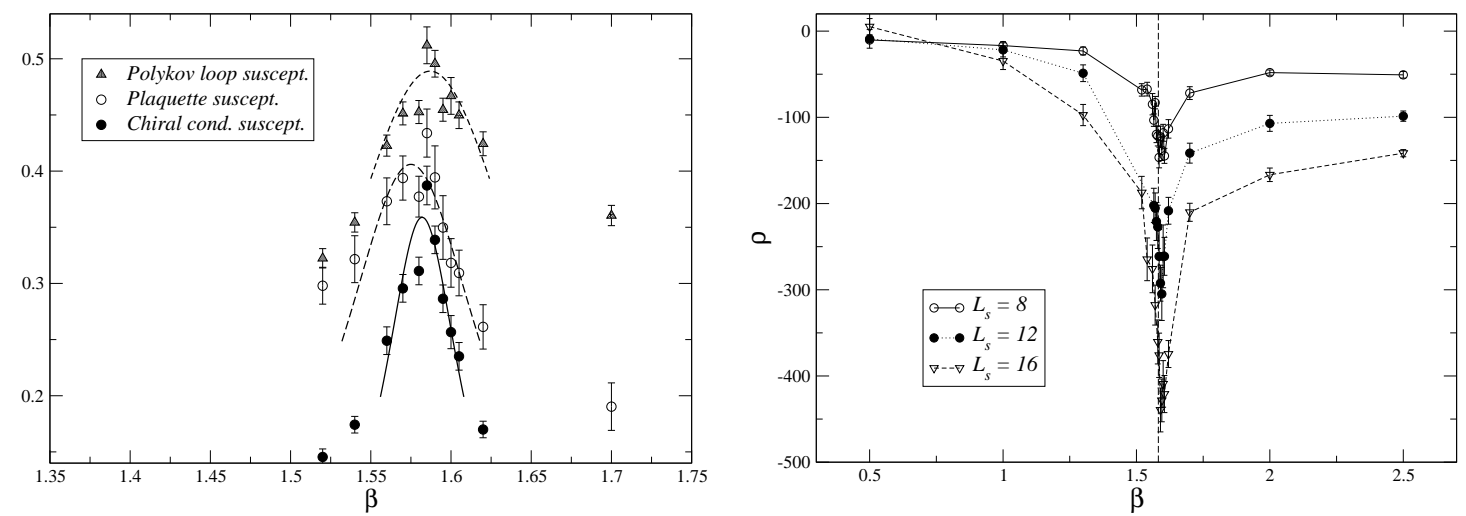

Figure 1: Plaquette, Polyakov and chiral susceptibilities on the $16^{3} \times 6$ lattice (normalized data) [left]. $\rho$ parameter [right].

know that $\langle\mathscr{M}\rangle \neq 0$ at $\hat{\mu}=0$ and we expect to meet a phase transition because the (pseudo)critical temperature lowers with increasing chemical potential. The lowest value of $T(\beta)$ examined, from a rough two-loop estimate of the $\beta$-function, corresponds to $T / T_{c}(\mu=0) \sim a(\beta=1.582) / a(\beta=$ $1.5) \sim 0.4$. In Fig. 2 [left] we show the chiral susceptibility on a $16^{3} \times 6$ lattice at $\beta=1.55$ and $\beta=1.50$. A fit locates the peak positions, that is the (pseudo)critical $\hat{\mu}$ corresponding to chiral restoration; we obtain $\hat{\mu}_{c}(\beta=1.50)=0.340(10)$ and $\hat{\mu}_{c}(\beta=1.55)=0.215(10)$. In Fig. 2 [right] we show instead $\rho_{D}$ as a function of $\hat{\mu}$ at the same values of $\beta$ and on various lattice sizes. For small chemical potentials $\rho_{D}$ is independent of the lattice size and practically vanishing, while at the chiral transition it has a sharp negative peak deeper and deeper as the spatial size is increased.
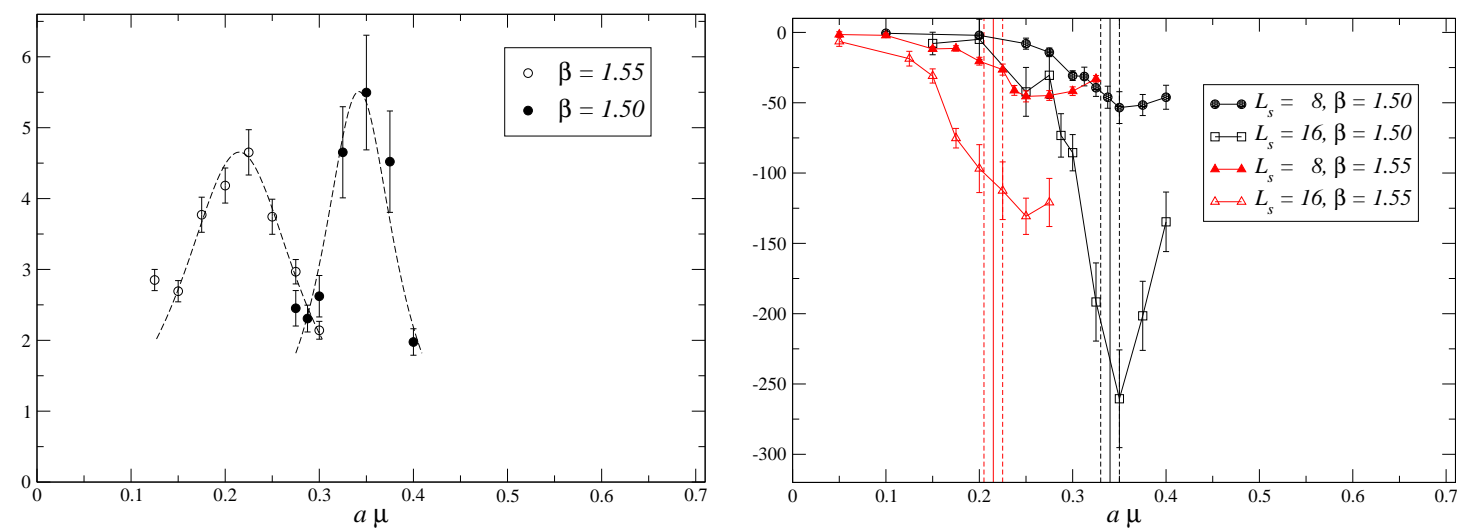

Figure 2: Chiral susceptibility peaks [left] vs $\rho_{D}$ peaks [right] at different $\beta$ 's. Vertical bands correspond to the $\mu_{c}$ derived from the chiral susceptibility.

\subsection{The transition line}

Having collected different locations of the deconfinement (chiral transition) line, we can fit the dependence $\beta_{c}(\mu)$ in the whole $\beta-\hat{\mu}$ plane. With a quadratic fit $\beta_{c}(\hat{\mu})=A+B \hat{\mu}^{2}$, we get $A=1.5828(16), B=-0.071(4)$ and $\chi^{2} /$ d.o.f. $=0.26$. The good value of $\chi^{2} /$ d.o.f. tells that a 
parabolic behavior fits well the critical line down to $T / T_{c} \sim 0.4$; indeed a quartic term $\hat{\mu}^{4}$ has a coefficient compatible with zero. The estimated location of the (pseudo)critical points is shown in Fig. 3 together with the fitted transition line.

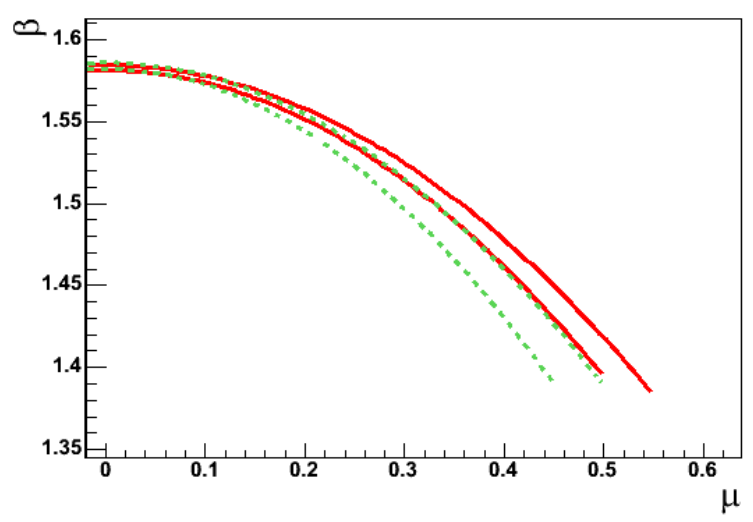

\begin{tabular}{|c|c|c|}
\hline$L_{s}$ & $\beta_{c}$ & $\mu_{c}$ \\
\hline $8^{3} \times 6$ & $1.584(2)$ & 0 \\
\hline $12^{3} \times 6$ & $1.587(2)$ & 0 \\
\hline $16^{3} \times 6$ & $1.582(2)$ & 0 \\
\hline $16^{3} \times 6$ & $1.568(2)$ & 0.15 \\
\hline $8^{3} \times 6$ & 1.55 & $0.222(10)$ \\
\hline $16^{3} \times 6$ & 1.55 & $0.215(10)$ \\
\hline $8^{3} \times 6$ & 1.5 & $0.325(10)$ \\
\hline $12^{3} \times 6$ & 1.5 & $0.349(15)$ \\
\hline $16^{3} \times 6$ & 1.5 & $0.342(10)$ \\
\hline
\end{tabular}

Figure 3: Chiral transition line (red) vs superconducting transition line (green) with error ranges [left] extracted from data of table [right].

The ratio $-\rho_{D} / \rho$ at the transition point has been used as an evaluator of the slope of the critical line (see Fig. 4), as claimed in Section 2: a good agreement with the slope of the fitted transition line in the $\beta-\hat{\mu}$ plane can be appreciated.
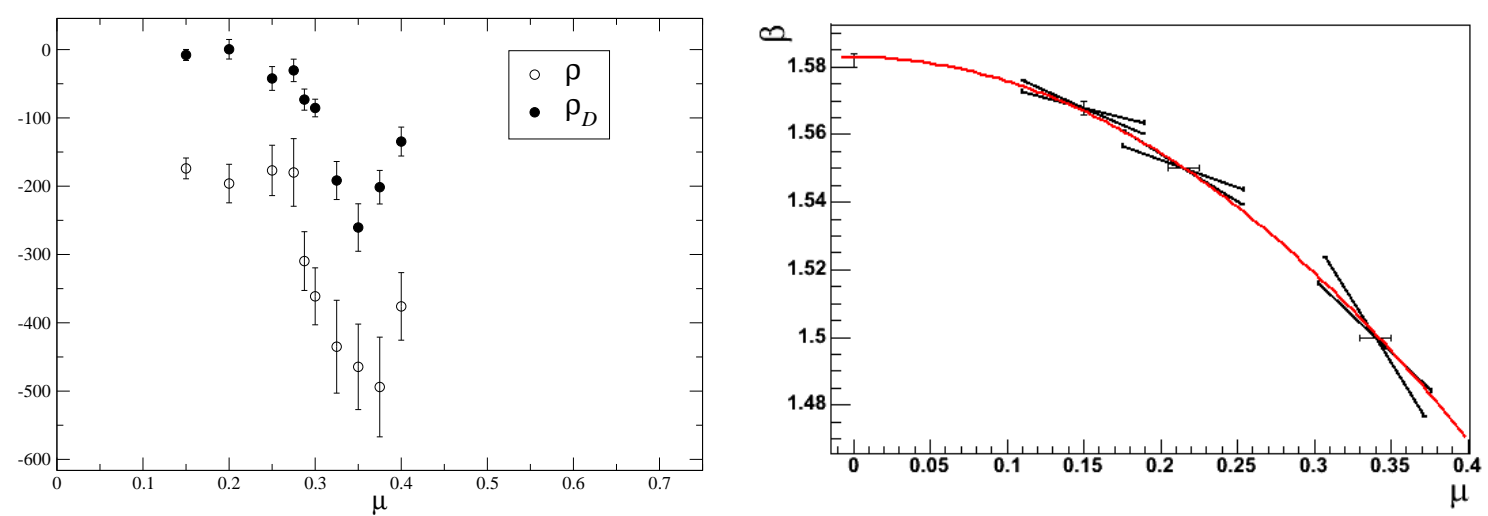

Figure 4: $\rho$ and $\rho_{D}$ at $\beta=1.5$ on a $16 \times 6$ lattice [left]. From this we compute $-\rho_{D} / \rho$ and draw the tangent on the transition line [right]. A nice agreement (within one standard deviation) can be appreciated

We can therefore draw two important conclusions from our results: dual superconductivity (confinement) disappears in presence of a critical density of baryonic matter; moreover the critical line in the $T-\mu$ plane corresponding to deconfinement coincides, at least within our present uncertainties, with the chiral transition line. 


\subsection{A few remarks on saturation}

Due to the Pauli exclusion principle, we cannot place more than one fermion with given quantum numbers per lattice site: when this limit is saturated fermion propagation gets quenched and the theory becomes equivalent to a pure gauge theory. Saturation is then an unphysical lattice artifact which may invalidate numerical results, and we need to be very careful in locating its onset.

We have looked to saturation effects at $\beta=1.55$. In Fig. 5 [left] we show the behaviour of some observables as a function of $\hat{\mu}$ up to $\hat{\mu}=1$.6. For $\hat{\mu}$ slightly above $\hat{\mu}_{c}$ the fermion density rises roughly with a cubic dependence in the chemical potential, as expected for a gas of free fermions, but then it saturates, departing from the cubic behaviour from $\hat{\mu} \sim 0.6-0.8$ on. Also the Polyakov loop stops rising at a similar value of $\hat{\mu}$, and then drops down; in the same region the plaquette falls to its quenched value. The full saturation is reached for $\hat{\mu} \sim 1.4-1.6$.

The susceptibilities of the disorder parameter (Fig. 5 [right]) display a positive unphysical peak at $\hat{\mu} \sim 0.7$ following the negative peak at $\hat{\mu} \sim 0.3$ which corresponds to the physical deconfinement transition. That means that the disorder parameter $\langle\mathscr{M}\rangle$, at first dropping to zero thus indicating deconfinement, then rises again due to saturation: actually the "saturation transition" leads to the $S U(2)$ pure gauge theory, which at $\beta=1.55$ and $L_{t}=6$ is confined.

Even if the saturation transition at $\hat{\mu} \sim 0.7$ is well separated from the physical transition at $\hat{\mu} \sim 0.3$, we notice that the "saturation line" in the $\beta-\hat{\mu}$ plane has a positive slope $\left(-\rho_{D} / \rho\right.$ from the saturation peaks of Fig. 5 [right] is greater than zero). That means that at lower $\beta$ the saturation transition could happen at lower values of $\hat{\mu}$ which added to the fact that the physical $\hat{\mu}_{c}$ with decreasing $\beta$, can easily cause an overlap of the two transitions, physical and unphysical, at lower values of $\beta$ (Fig. 6).

Therefore one should be careful about the possible effects of Pauli blocking on the study of finite density QCD in the strong coupling regime. The warning is general even if the situation may depend on the temporal extent $L_{t}$ of the lattice as well on the number of flavors and colors.
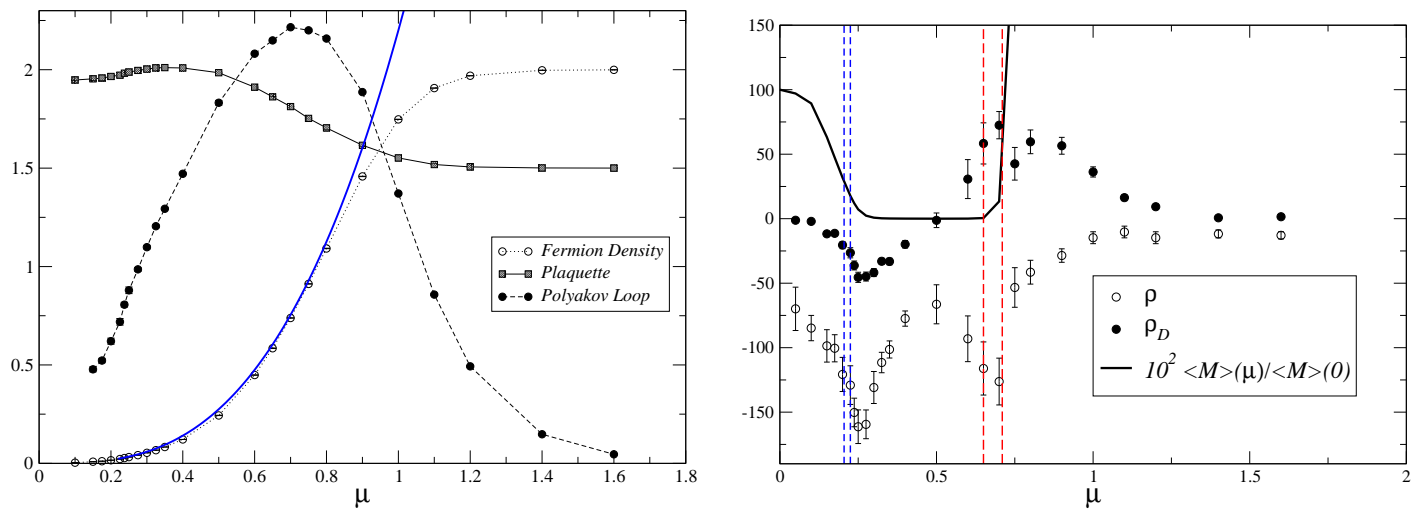

Figure 5: Effects of Pauli blocking: as the fermion number saturates different observables indicate the onset of a "quenched" phase [left]. $\rho$ and $\rho_{D}$ individuate the unphysical transition with peaks of different sign $\left(-\rho_{D} / \rho>0 \Rightarrow\right.$ positive slope for the critical line) [right]. 

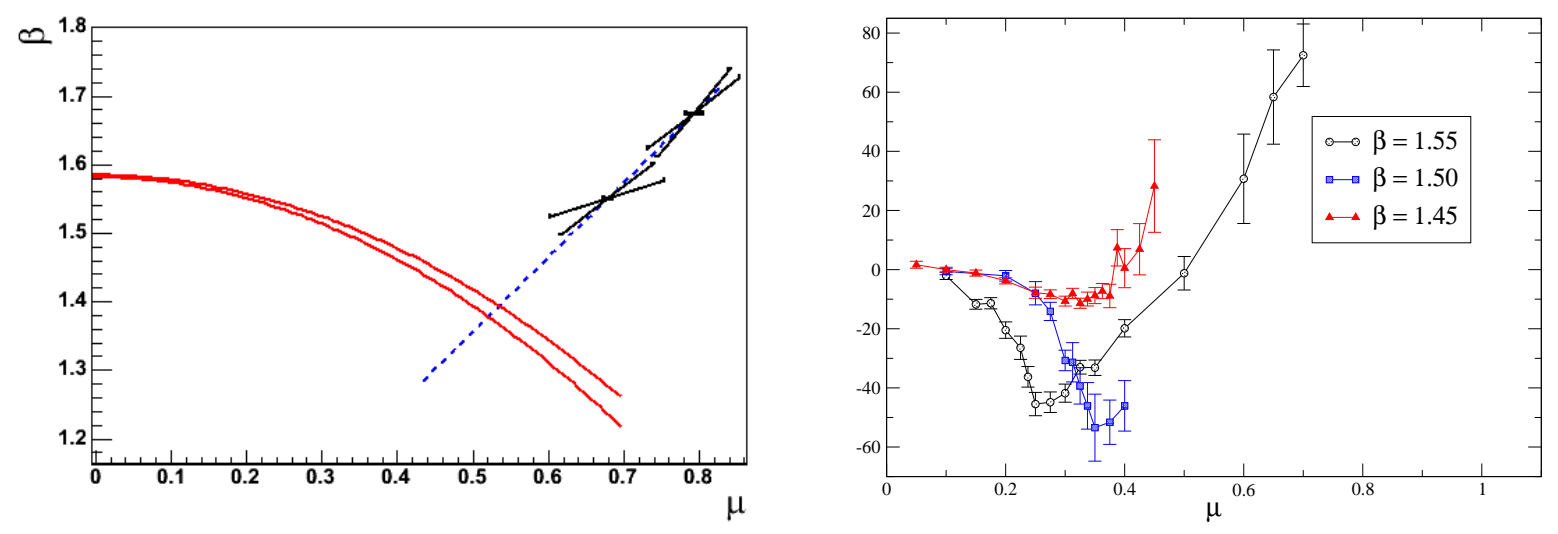

Figure 6: Physical transition line (continuous) vs unphysical saturation line (dashed) [left]: at $\beta=1.45$ the saturation prevents a deep peak for $\rho_{D}$ to form as for greater values of $\beta$ [right].

\section{Conclusions}

We have shown how a finite density of baryonic matter induces deconfinement and that, in the region explored, the deconfinement transition coincides with chiral symmetry restoration also at $\mu \neq 0$. Furthermore we have given a general warning on the possible effect of Pauli saturation on the study of the QCD phase diagram at low $\beta$.

\section{References}

[1] S. Hands, S. Kim and J. I. Skullerud, hep-lat/0604004.

[2] L. McLerran and R. D. Pisarski, arXiv:0706.2191

[3] M. D'Elia and M. P. Lombardo, Nucl. Phys. B 752 (2006) 124 [hep-lat/0602022]; Phys. Rev. D 70 (2004) 074509 [hep-lat/0406012].

[4] G. 't Hooft, High Energy Physics, in proceeding of EPS International Conference, Palermo 1975

[5] S. Mandelstam, Phys. Rept. 23 (1976) 245.

[6] G. Parisi, Phys. Lett. B 60 (1975) 93.

[7] L. Del Debbio, A. Di Giacomo and G. Paffuti, Phys. Lett. B 349 (1995) 513 [hep-lat/9403013].

[8] A. Di Giacomo and G. Paffuti, Phys. Rev. D 56 (1997) 6816 [hep-lat/9707003].

[9] A. Di Giacomo, B. Lucini, L. Montesi and G. Paffuti, Phys. Rev. D 61 (2000) 034503 [hep-lat/9906024]; Phys. Rev. D 61 (2000) 034504 [hep-lat/9906025].

[10] J. M. Carmona et al. Phys. Rev. D 64 (2001) 114507 [hep-lat/0103005].

[11] J. M. Carmona et al. Phys. Rev. D 66 (2002) 011503 [hep-lat/0205025].

[12] M. D’Elia et al. Phys. Rev. D 71 (2005) 114502 [hep-lat/0503035].

[13] S. Conradi, A. D'Alessandro and M. D’Elia, Phys. Rev. D 76 (2007) 054504 [arXiv:0705.3698]. 